\title{
63. Jahrestagung der Deutschen Gesellschaft für Phlebologie, 8.-11. September 2021 in Aachen
}

Mit dem Kongressthema „Aachen 2021 Synergie und Vielfalt" fand die 63. Jahrestagung der Deutschen Gesellschaft für Phlebologie vom 8.-11. September 2021 im Eurogress in Aachen statt ( $\triangleright$ Abb. $\mathbf{1})$.

Das in den vergangenen Jahren bewährte Konzept des „aktiven wissenschaftlichen Beirats" hat sich auch in diesem Jahr wieder einmal bewährt. Die Organisation einzelner wissenschaftlicher Sitzungen in den jeweiligen Spezialgebieten der Vorsitzenden hat dazu beigetragen, dass ein breit gefächertes Programm gestaltet werden konnte ( $\triangleright$ Abb.2). Mal waren die Sitzungen von Kurzreferaten geprägt, mal waren Sitzungen von Grundsatzreferaten gekennzeichnet, die den wissenschaftlich aktuellen Status quo eines Themas präsentierten. Nach der langen Corona-bedingten Kongresspause haben zahlreiche internationale Referenten den Weg nach Aachen gefunden. In simultan übersetzten Sitzungen kamen so nationale und internationale Teilnehmer auf ihre Kosten. Darüber hinaus hatten auch die „Daheimgebliebenen“ die Mög- lichkeit, über das Livestream-Angebot am Kongress teilzunehmen.

Der Mittwoch stand ganz im Zeichen von Crash-Kurs und Update-Phlebologie, organisiert von Frau PD Dr. Felizitas Pannier und Prof. Tobias Görge. Wie in den letzten Jahren zeigte sich hier, dass gerade dieses Format viele neue und junge Gesichter anspricht. Die nachhaltigen Diskussionen, die verschiedenen interdisziplinären Blickwinkel und Sichtweisen, gerade auch aus der Praxis, bereichern beide Kurse immens.

Der Eurogress in Aachen war in Corona-Zeiten eine ideale Location: kurze Wege, die das Zusammenspiel von Fortbildungsblöcken und den Besuch der Industrieausstellung förderten. Das große Interesse des phlebologischen Nachwuchses freut uns als Gesellschaft ungemein.

Im Weiterbildungsforum, organisiert von Dominic Mühlberger und Jasmin WoitallaBruning, wurden Grundlagen in Diagnostik und Therapie in der Phlebologie vermittelt, es wurden berufspolitische Fragen ange-

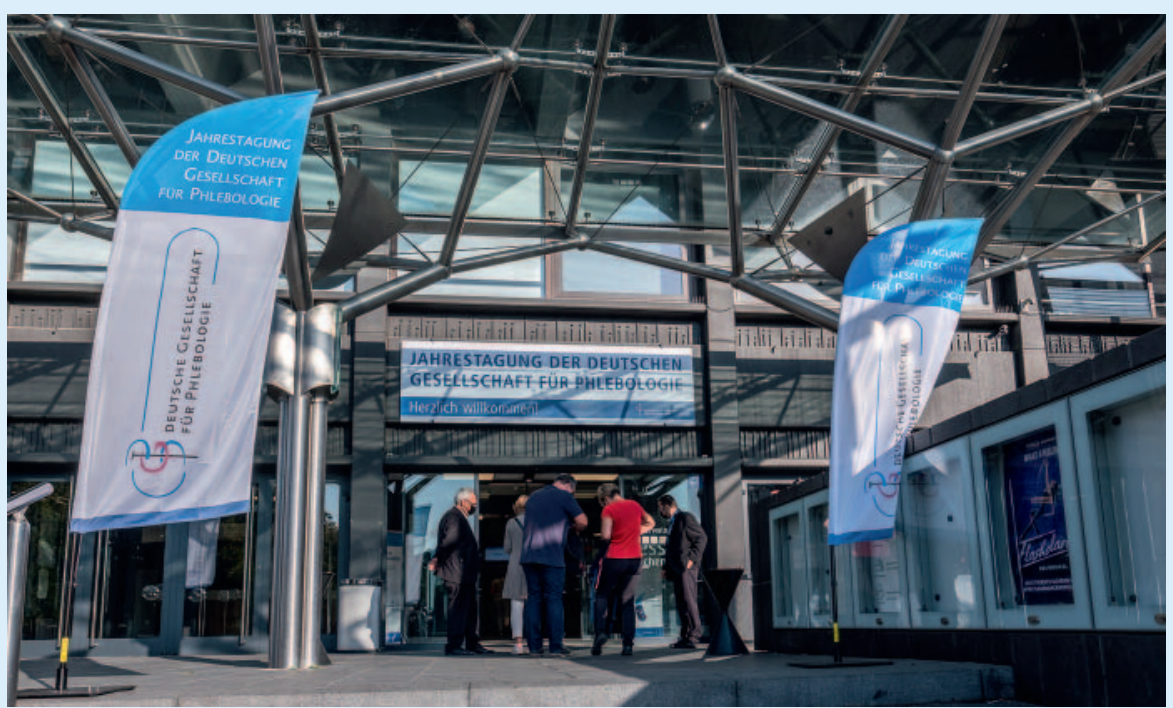

- Abb. 1 Eurogress Aachen. Quelle: T. Hauss/Deutsche Gesellschaft für Phlebologie [rerif]. sprochen und wieder praxisrelevante „Fälle auf die Schnelle“ vorgestellt.

An allen 3 Kongresstagen waren die ,ask the experts"-Sitzungen früh um 8 Uhr gut besucht. Die Möglichkeit, Experten zu allen Themen, die Phlebologen in der Praxis beschäftigen, persönlich zu befragen und vor allem über eigene Erfahrungen und Fälle zu diskutieren, wurde gut angenommen.

Parallel zum wissenschaftlichen Programm wurden für interessierte Teilnehmer zahlreiche Hands-on-Workshops angeboten. Neben den Klassikern Sklerotherapie und Duplexsonografie waren in diesem Jahr z. B. auch Workshops zur operativen Therapie der Varikose, zum Venous Stenting oder zur Thrombose im Angebot.

Darüber hinaus wurde den täglichen Problemen des Praxisalltags reichlich Platz ein-

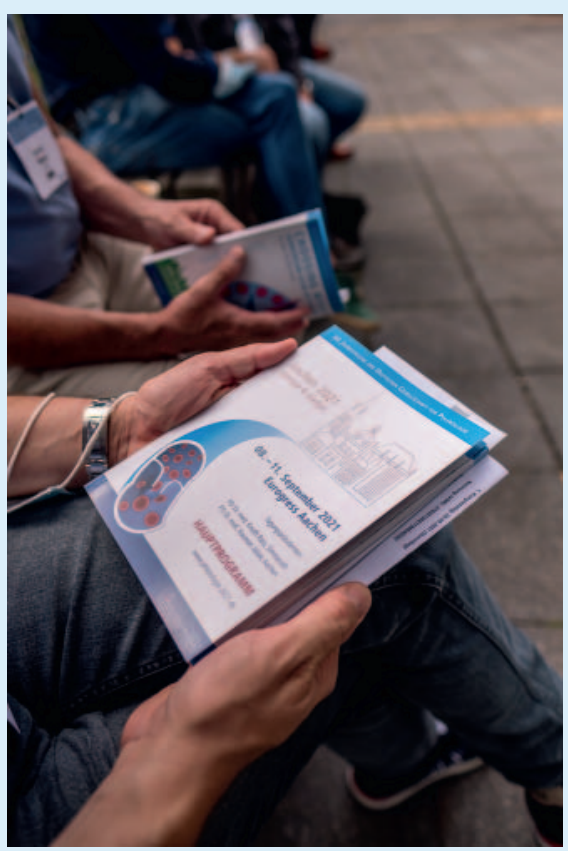

- Abb. 2 Programmauswahl. Quelle: T. Hauss/Deutsche Gesellschaft für Phlebologie [rerif]. 
geräumt: so gab es wissenschaftliche Sitzungen zum Themenfeld Kompression, die Sklerosierungstherapie wurde behandelt und der große Raum der operativen und endoluminalen Therapie fand in zahlreichen Sitzungen Berücksichtigung. Venenerhaltende Therapiekonzepte bei der Stammvarikose und das tiefe Venensystem konnten erläutert werden, ebenso wie die Thrombosetherapie. Im „Interessanten Fall“ wurden Situationen aus dem Praxis- und Klinikalltag geschildert und ausführlich diskutiert.

55 unterstützende/ausstellende Firmen konnten die Innovationen in der fachgebundenen Medizintechnik und der Pharmakologie begleiten und so unseren Besuchern auch auf diesem Gebiet aktuelles Wissen vermitteln.

Wie immer stellte der Kongress die Plattform für Ehrungen und Preisverleihungen dar. Die Preisträger wurden in der letzten Ausgabe der Phlebologie ausführlich vorgestellt.

Der gesamte Kongress fand in einer lockeren, fröhlichen und gastfreundlichen Grundstimmung statt - wir alle haben uns gefreut, wieder einmal persönlich miteinander in Kontakt zu treten.

Zum Abschluss noch der herzliche Dank an alle, die zum Gelingen der 63. Jahrestagung der DGP beigetragen haben - dem wissenschaftlichen Beirat, den aktiven Referenten und Vorsitzenden, unserer tollen Band am Festabend, der unterstützenden Industrie, ohne die eine solche Veranstaltung nicht möglich wäre, den Musikern des Rahmenprogramms, den vielen hilfsbereiten und freundlichen Hostessen, der Technik, der Kongressorganisation und dem zuarbeitenden Service, und vor allem den Kongresspräsidenten PD Dr. Knuth Rass und PD Dr. Houman Jalaie.

\section{Jahrestagung der Deutschen Gesellschaft für Phlebologie}

\section{CHOOSING WISELY GEMEINSAM KLUG ENTSCHEIDEN}

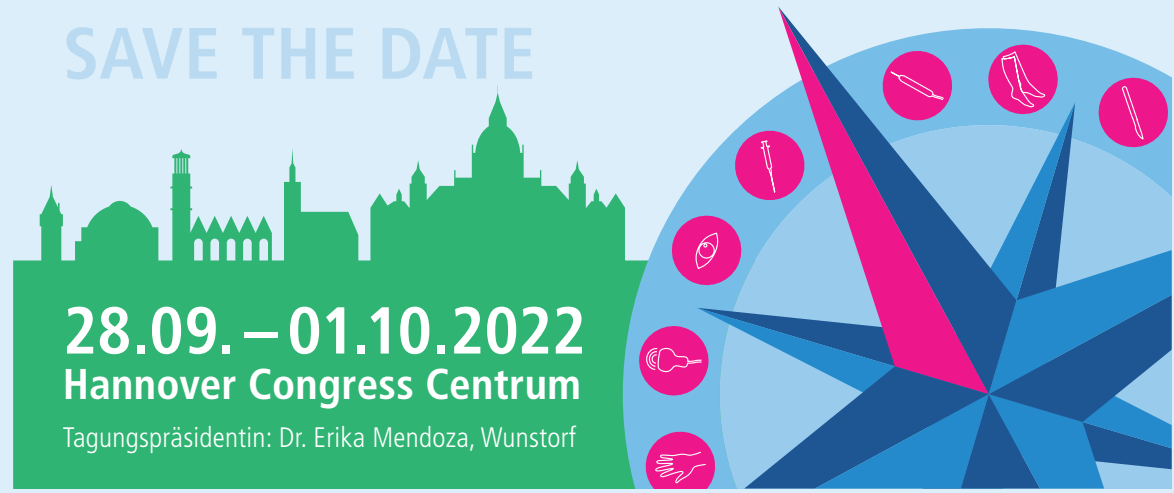

- Abb. 3 Choosing wisely - Kongressmotto in Hannover [rerif].

\section{Choosing Wisely - 28.09.-} 1.10.2022

Nun schauen wir nach Hannover - vom 28.09.-1.10.2022 freuen wir uns im Congress Centrum in Hannover zu Gast zu sein. Die Veranstaltung steht unter dem Thema „Choosing Wisely in der Phlebologie" mit Dr. Erika Mendoza als unserer Kongresspräsidentin ( $\triangleright$ Abb. 3 ).

Immer wieder aktualisierte Informationen zu unserer Tagung erhalten Sie auf https:// phlebologie-2022.de. Hier haben Sie auch bereits die Möglichkeit, Ihre Abstracts einzureichen.

Im Rahmen der Tagung werden neben wissenschaftlichen Sitzungen wieder zahlreiche Hands-on-Workshops angeboten, so z. B. Ultraschallkurse der oberflächlichen und tiefen Beinvenen, Workshops zur Sklerotherapie und zur endoluminalen Behandlung, darüber hinaus werden Kurse in chirurgischen Techniken angeboten.
Mit einem Update Phlebologie für Gynäkologen und Orthopäden \& Unfallchirurgen sowie einem Hausärztetag soll das vor Ort versammelte „Wissen“ den Kollegen aus angrenzenden Regionen angeboten werden. Es wird gemeinsame Sitzungen mit den Gesellschaften für Lymphologie und Gefäßchirurgie geben. Am Freitag bieten wir erneut eine ICW-Rezertifizierungsveranstaltung für Pflegende zum Thema „Chronische Wunden" an.

Das Hannover-Congress-Centrum liegt in der Stadt und trotzdem im Grünen, und ist bekannter Treff- und Mittelpunkt für das gesellschaftliche und kulturelle Leben der Landeshauptstadt Hannover.

\section{IMPRESSUM}

Verantwortlich für Mitteilungen der DGP:

Dr. med. Erika Mendoza 